\title{
Hypothesis: mPGES-1-derived Prostaglandin E2, a so far missing link in COVID-19 pathophysiology?
}

Jan Smeitink ${ }^{1,2}$, Jiang $\mathrm{X}^{1,2}$, Pecheritsyna $\mathrm{S}^{2}$, Renkema $\mathrm{H}^{2}$, Rob van Maanen ${ }^{2}$, Julien Beyrath ${ }^{2}$

${ }^{1}$ Department of Pediatrics, Radboud Center for Mitochondrial Medicine, Radboudumc, Nijmegen, The Netherlands, ${ }^{2}$ Khondrion B.V., Nijmegen, The Netherlands

Corresponding author:

Jan A.M. Smeitink, MD, PhD, MAE

Khondrion BV

Philips van Leydenlaan 15

6525EX Nijmegen

The Netherlands

E-mail: smeitink@khondrion.com

Keywords: COVID-19, prostaglandins, mPGES1 inhibitor, ARDS, sonlicromanol, KH176 


\begin{abstract}
With frequencies varying up to $20 \%$, treatment resistant pulmonary failure is a major life-threatening complication in COVID-19 (SARS-CoV-2, HCoV19) disease pathology. Both acute respiratory distress syndrome (ARDS), proposed to be caused by an over-reacting immune system which floods the lung with edema, a liquid consisting of inflammatory cells, and diminished lung perfusion, have been postulated to cause this treatment resistant lung failure. Aging, co-morbidities, male gender and obesity are pre-existing factors associated with the more severe outcome. Thrombosis is more frequently observed than usually seen during ICU admission. Different hypotheses explaining the pathophysiological cascade leading to fast progressing severe COVID-19 disease and how to counteract it have been proposed. A variety of intervention studies to control severity are ongoing or planned. Not suggested so far, we here hypothesize that the inflammatory lipid modulator prostaglandin $E_{2}$ $\left(\mathrm{PGE}_{2}\right.$ ) executes a prominent role in COVID-19 pathophysiology. Based on this we suggest measuring $\mathrm{PGE}_{2}$ in patients and evaluating selective inhibition of the human microsomal prostaglandin $E$ synthase1 (mPGES-1) as a potential innovative therapeutic approach in this devastating condition for which sonlicromanol, a drug currently in phase $2 \mathrm{~b}$ studies for mitochondrial disease, is a candidate.
\end{abstract}

\title{
Introduction
}

The recent burst of COVID-19 infections with its high pulmonary related mortality figures has shocked the world and warrants global efforts in developing new treatment strategies (Guo et al., 2020; Martine et al., 2020; Rodriguez-Morales et al., 2020)

To enter the target cell COVID-19 binds, via spike-glycoproteins expressed on its envelope, to angiotensin-converting enzyme 2 (ACE2) (Chen et al., 2020). Coughing, sneezing and even talking spread the virus of an infected subject through the air after which it may enter the body via the eyes, mouth or nose in a new host. Expression of ACE2 is ubiquitous with relatively high expression in respiratory epithelial cells, type I and II alveolar cells and the intestines(Harmer et al., 2002; Xu. et al., 2020). Members of the Human Cell Atlas consortium recently reported that the COVID-19 entry genes (ACE2 and the protease TMPRSS2) are most highly expressed in nasal goblet and ciliated cells within human airways (Sungnak et al., 2020). Mucosal mast cells in the nasal cavity and the submucosal respiratory tract act as primary lines of defense (Kritas et al., 2019).

Upon viral invasion, depending on the type of cells targeted, different cellular responses may occur (Figure 1). These include viral cell death mechanism as well as early and late inflammatory processes.

After viral infection, nasal goblet and ciliated cells undergo cell death (apoptosis, necrosis, pyroptosis) releasing pathogen-associated molecular patterns (PAMPs) and damage-associated molecular patterns (DAMPs) to activate the innate immune response. This activation involves recruitment of granulocytes to the injured tissue and the release of inflammatory mediators, including proinflammatory cytokines such as TNF- $\alpha, \mathrm{IL}-1 \beta$ and IL- 6 and lipid mediators such as prostaglandins (PGs and leukotrienes (LTS). Both the proinflammatory cytokines and the lipid mediators evoke an acute inflammatory process (hours to days) to clear the pathogens and damaged tissues. Acute inflammation is resolved, and the tissue is repaired when PAMPs, DAMPs, pathogens and damaged tissues are cleared. Granulocyte recruitment ends with a down-regulation and scavenging of chemokines, and 
recruited granulocytes are subsequently cleared by efferocytosis (apoptotic cells released by phagocytic cells)(Yao and Narumiya, 2019).

Invasion of the mucosal mast cells in the nasal cavity and submucosal respiratory tract on the contrary cause an early inflammatory response with the release of histamine and proteases followed by a late inflammatory response releasing pro-inflammatory IL-1 family members (IL-1, IL-6 and IL-33).

COVID-19 invasion and the responses of the different cells of the respiratory tract have led to progressive therapy resistant inflammation, as seen in an important number of infected patients. Older age, comorbidities, male gender and obesity are somehow related to a poorer outcome. So far, the results of therapeutic interventions like the anti-malaria drugs chloroquine, hydroxychloroquine and others show conflicting results (Ferner and Aronson, 2020). Tens of thousands of deaths have already been reported. These unsatisfactory outcomes urgently call for alternative approaches. Following a short overview of recently initiated international trials, we here summarize the potential diseasemodifying role the lipid mediator prostaglandin E2 may have in COVID-19 pathology. Based on this we call for the measurement of $\mathrm{PGE}_{2}$ in affected patients and propose selective inhibition of the microsomal prostaglandin E synthase-1 as a potential new treatment approach in preventing patients from severe disease progression and from death.

\section{Initiated international trials}

Scientists, industry and politicians are working closely together to control viral spread and to develop potential treatments for COVID-19. A variety of measures and clinical trials are ongoing, so far with disappointing results with respect to mortality. Obviously, much efforts concentrate on avoiding spread of the virus, epidemiology and the development of diagnostic kits and vaccines, as well as highthroughput studies evaluating the repurposing potential of drugs on the market counteracting e.g. viral replication. The latter leading to a more or less immediate drug availability. Alternatively, drug compounds under development are also being evaluated for their potential to counteract the COVID19 disease burden. Here, we will address an example of the latter approach.

The World Health Organization just launched the Solidarity Trial Initiative (www.who.int), an international clinical trial to help find an effective treatment for COVID-19. The Solidarity trial will compare four treatment options (Box 1) against standard of care, to assess their relative effectiveness against COVID-19. By enrolling patients in multiple countries, the Solidarity trial aims to rapidly discover whether any of the drugs slow disease progression or improve survival.

With recruitment starting April $7^{\text {th }}, 2020$, the European Discovery trial has started (Box 2). This trial plans to include 3,200 European patients from Belgium, France, Germany, Luxembourg, the Netherlands, Spain, Sweden, and the United Kingdom (www.presse.inserm.fr). Allocation of patients to the various treatment modalities will be randomized, i.e. by random draw, but patients and physicians will know which treatment is used (this is called an open trial).

Both initiatives have indicated that other drugs can be added based on emerging evidence and numerous companies are exploring this possibility. 


\section{COVID-19: clinical observations and pathophysiology}

In a short time frame large numbers of patients allocated to ICUs have revealed some peculiar characteristics present in the most severely affected patients. To these belong: a) COVID-19 is more severe in the elderly population, b) the number of males in ICUs far exceeds that of females, c) the number of obese patients exceeds those of the lean population and d) next to the severe lung-related problems, thrombosis is seen more frequently than expected in ICUs.

We combined these observations, together with the pre-existing knowledge gathered during this and earlier viral pandemics, into a new pathophysiological hypothesis, that "lipid mediators play a crucial role in COVID-19 disease progressing and are amenable to treatment intervention". Sharing of the concept to the scientific and medical community enables it to be challenged. If supported, preclinical research and/or exploratory trials might be initiated and we appreciate submission of proposals.

We postulate human prostaglandin $E_{2}\left(P E_{2}\right)$ is an important factor contributing to COVID-19 hyperinflammatory and immune responses. We suggest evaluating whether specific lowering of $\mathrm{PGE}_{2}$ via selective inhibition of the human microsomal prostaglandin E synthase-1 (mPGES-1), while not having effects on other prostaglandins (Figure 1), might positively influence patient mortality outcome of ARDS-related COVID-19.

\section{$\mathrm{PGE}_{2}$ in inflammation}

Prostaglandins (PGs) are a group of physiological lipid mediators (Gomez et al., 2013; Tsuge et al., 2019). They not only show diverse hormone-like effects but also participate in pathological reactions, such as inflammatory and nociceptive responses (Miller, 2006). PGs are synthesized in the cell from arachidonic acid (AA) via phospholipase- $\mathrm{A}_{2}$. Next, the cyclooxygenases, (COX-1 and COX-2), convert AA into prostaglandin $\mathrm{H} 2\left(\mathrm{PGH}_{2}\right)$. Finally, $\mathrm{PGH}_{2}$ is further metabolized into four major biologically active PGs ( $\mathrm{PGD}_{2}, \mathrm{PGI}_{2}, \mathrm{PGE}_{2}$, and $\mathrm{PGF}_{2 \alpha}$ ) and thromboxane $\mathrm{A} 2$ ( $\mathrm{TXA}_{2}$ ), which are synthesized by cell- and tissue-specific synthases and isomerases(Park et al., 2006; Tron et al., 2006; Nakanishi et al., 2010).

Elevated levels of $\mathrm{PGE}_{2}$ have been ascribed to play an essential role in inflammation. $\mathrm{PGE}_{2}$ is generated from the action of three different prostaglandin E synthases on PGH2, microsomal isoforms (mPGES-1 or mPGES-2) or cytosolic PGES (cPGES) (Hara et al., 2010) (Figure 1). To date, mPGES-1 emerges as a key enzyme in the formation of $\mathrm{PGE}_{2}$ in inflammatory states, as it is up-regulated by various inflammatory stimuli, such as LPS or IL-1 $\beta$ while CPGES and MPGES-2 are known to be constitutively expressed in many organs and tissues (Riendeau et al., 2005; Ikeda-Matsuo et al., 2006; Smith et al., 2011).

Today mPGES-1 is considered as a potential safer target for anti-inflammatory drugs since it is only expressed in diseased tissues and downstream of the COX enzymes (Ding et al., 2018). COX enzymes are the current target of most commercially available non-steroidal anti-inflammatory drugs (NSAIDs, COX inhibitors). But their use can be limited by their non-specific inhibitory effects on all prostanoids downstream of $\mathrm{PGH}_{2}$, which lead to gastric side effects or increased risk of cardiovascular morbidity and mortality. Additionally, selective inhibition of mPGES-1 would still allow basal production of $\mathrm{PGE}_{2}$ via its two other constitutive enzymes CPGES and mPGES-2 involved in physiological role of $\mathrm{PGE}_{2}$. 


\section{Linking COVID-19 clinical observations and PGE $_{2}$}

As stated, gender, ageing and obesity have been noticed to be pre-existing severity factors. It has earlier been noticed that the severity and course of inflammatory process differ between women and men. Simona Pace and colleagues (Pace et al., 2017) found that freshly isolated and immediately LPS stimulated human neutrophils from males produced more $\mathrm{PGE}_{2}$ than cells from females and that this might be caused by increased COX-2 expression and by shunting phenomena due to suppressed leukotrienes formation. They conclude that the observed sex-difference might help to explain, at least in part, the sex dimorphism in innate immune disorders such as sepsis and post-surgery infections. Here, we suggest that the increased $\mathrm{PGE}_{2}$ response in males, being an important lipid mediator, may be one causative factor explaining the more severe disease state in males following COVID-19 infection. Measurements of $\mathrm{PGE}_{2}$ in males and females and correlating these with disease severity in COVID-19 seems warranted.

With a few exceptions, children seem to be less prone to develop severe COVID-19 than the aged population. Several explanations such as their young immune system, ACE2 receptor levels and exposure to other corona viruses have been put forward. Another possible explanation is the aging lung environment (Zhao et al., 2011; Angelidis et al., 2019). The Perlman group showed in mice infected with respiratory viruses that increases in $\mathrm{PGD}_{2}$ upon aging correlated with progressive impairment in respiratory DC migration resulting in diminished $T$ cell responses and more severe clinical disease in older mice. They suggest that inhibiting prostaglandins, in this case $P G D_{2}$, may be a useful approach to enhance the host antiviral response (Zhao et al., 2010, 2011). Age-related increase of $\mathrm{PGE}_{2}$ was also reported in mouse macrophages (Wu et al., 1998) and, since it is known to inhibit the immune system (Kalinski, 2012), pre-existing increased $\mathrm{PGE}_{2}$ level might also cause higher sensitivity to COVID-19.

Next to mechanical issues, obesity is accompanied by a systemic, chronic low-grade inflammation as well as dysfunctions of several innate and adaptive immune cells. Recent findings emphasize an impaired functionality and phenotype of natural killer (NK) cells under obese conditions (Bähr et al., 2020). Elevated circulating levels of $\mathrm{PGE}_{2}$ have been observed in obese individuals (Fain et al., 2002; Pawelzik et al., 2019). Furthermore, IL-1ß stimulates the production of $\mathrm{PGE}_{2}$ primarily by transcriptionally upregulating $\mathrm{COX} 2$ through the action of the transcription factor NF-kB (Osborn et al., 2008).

More than expected, thrombosis has been observed in the severely affected COVID-19 subjects. The underlying reason of thrombosis might obviously be related to longer term immobility while being on ventilators. Other causes though may exist. In line with our hypothesis it was shown that $\mathrm{PGE}_{2}$, through its platelet EP3 receptor, is a central actor in intravascular thrombosis (Gross et al., 2007).

\section{ARDS, viral and bacterial infections and NSAIDs}

Since its first description in 1967 and despite numerous therapeutic interventions the mortality of ARDS still remains high. Examples include two double-blind RCTs in ventilated ARDS patients suggesting that antioxidants show no benefit and actually may be detrimental (together with omega3 fatty acid and gamma-linolenic acid (Rice et al., 2011), and as monotherapy (Morris et al., 2008). 
Another therapeutic intervention includes the use of NSAIDS downregulating the activity of cyclooxygenase 1 (COX1, Figure 1). A recent meta-analysis of aspirin in patients at risk of ARDS showed no reduction in mortality but preventive administration could reduce the risk for ARDS (Yu et al., 2018). One important limitation of the study was that subgroup analysis on the potential origin of the ARDS could not be made. One problem with the usage of COX1 (NSAIDs) and COX2 inhibitors (COXibs), is the a-selectivity with respect to all PG and Tromboxane $A_{2}$ downregulation. Agard et al. (2013) reviewed the role of $P G E_{2}$ in bacterial infection and concluded that inhibition of $\mathrm{PGE}_{2}$ may represent a therapeutic way to treat bacterial infections (Agard et al., 2013).

\section{Viral infection, ARDS and PGE 2}

$\mathrm{PGE}_{2}$ is known to have immunomodulatory roles (Kalinski, 2012) and, more particularly in the context of respiratory viral infection (McCarthy and Weinberg, 2012). The modulatory role of $\mathrm{PGE}_{2}$ in several viral infections has recently been reviewed and both inhibitory and activating effects of $\mathrm{PGE}_{2}$ on various immune system pathways identified (Sander et al., 2017). In most of the viral infections studied, $\mathrm{PGE}_{2}$ was reported to increase viral pathogenicity (HSV, CMV, EBV, RV, CVB3, EV71, PSaV, VSV, LCM, RSV, and HTLV-III), which occurred by affecting not only the host immunity, but also viral transcription, translation and/or replication. In the case of $\mathrm{CMV}$ infection $\mathrm{PGE}_{2}$ was shown to support viral replication independently of its role on host immunity (Mocarski, 2002). Consequently, selectively decreasing $\mathrm{PGE}_{2}$ might counteract viral pathogenicity. However, in the case of PIV3 and HBV, PGE 2 was shown to have both a stimulatory and an inhibitory role on viral pathogenicity. No data about $\mathrm{PGE}_{2}$ effects in corona viruses was presented in this review. Based on the results of one of the scarce in-vivo studies performed so far, in this case in an influenza A virus (IAV) infection mouse model, selective inhibition of $\mathrm{mPGES}-1$ has been proposed as a therapeutic option to enhance antiviral immunity. Indeed, mPGES1 knockout mice infected with influenza A virus had lower viral titers in the lung and reduced morbidity compared to wild-type mice (Coulombe et al., 2014; Full and Gack, 2014). The authors showed that $\mathrm{PGE}_{2}$ was increased in lungs of wildtype mice shortly after IAV infection and that $\mathrm{PGE}_{2}$ inhibits important function of alveolar macrophages, a central player in host immune response against respiratory virus. Interestingly, the treatment of mice infected with IAV, even three days after infection, with inhibitors of mPGES-1 significantly improved the survival of the animals. Further mPGES-1 inhibitors with anti-influenza activities were identified and shown to act via the inhibition of $\mathrm{PGE}_{2}$ production (Park et al., 2016). Overall, these results indicate that selective inhibition of MPGES-1 could enhance the host immune response against COVID-19. Finally, we hypothesize the age- or proinflammatory disease-related increases in $\mathrm{PGE}_{2}$ might underline the higher severity of COVID-19 in aged and obese populations.

It is important to note that several of the downstream products of the $\mathrm{PGE}_{2}$ pathway are required for normal physiological functioning (Sander et al., 2017). Additionally PGE2 had been shown to have both stimulatory and inhibitory effect on the immune system depending on the targeted cell type, inflammatory or physiological context or local concentrations. For instance, low (nanomolar) concentration of PGE2 were shown to activate T-cells while high (micromolar) were shown to inhibit the immune response (Sreeramkumar et al., 2012). This is where selective inhibition of mPGES-1 brings a crucial advantage over for instance upstream COX1/2 inhibition as it is mainly expressed in inflammatory context and responsible for the high production of $\mathrm{PGE}_{2}$ and would still allow basal biosynthesis of $\mathrm{PGE}_{2}$ by the two other constitutive synthases (cPGES and mPGES-2). 


\section{Selective mPGES-1 inhibition to attenuate COVID-19 associated ARDS: a hypothesis to challenge}

Human mPGES-1 is recognized as a promising target for the next generation of anti-inflammatory drugs, without the side effects of those currently available (Ding et al., 2018). Our hypothesis, that selective mPGES-1 inhibition might attenuate COVID-19 associated disease symptoms, e.g. ARDS, and thereby prevent ICU admissions and decrease mortality, is based on the role $\mathrm{PGE}_{2}$ has as an inflammatory modulator in viral infections and further literature supporting that targeted $\mathrm{PGE}_{2}$ inhibition enhances antiviral immunity (Ricciotti and Fitzgerald, 2011; Coulombe et al., 2014; Bergqvist et al., 2020) (Figure 2). That $\mathrm{PGE}_{2}$ belongs to the many factors contributing to the uncontrolled inflammation observed in obese individuals adds to our hypothesis (González-Périz and Clària, 2010; Pierre et al., 2018). Besides, mPGES-1 knock-out mice have been shown to reduce diet-induced lowgrade inflammation and adiposity (Pierre et al., 2018).

Findings on the role of $\mathrm{PGE}_{2}$ in various models of pulmonary inflammation in preclinical models are mixed. Injection of oleic acid (OA) into rats results in an ARDS-like syndrome inducing acute increases of $\mathrm{PGE}_{2}$ as well as leukotrienes and endothelins (Pritze et al., 1992). In mice, ARDS induced by OA resulted in lung inflammation and edema and increases in $\mathrm{PGE}_{2}$ and leukotriene B4 (Gonçalves-DeAlbuquerque et al., 2013). In rats administered LPS to induce ARDS, parecoxib reduced levels of $P_{G_{2}}$, tumor necrosis factor- $\alpha$, interleukin- $1 \beta$ and other inflammatory markers, and improved lung histopathology and survival (Meng et al., 2017).

Limitations of these findings result from the uncertainty over whether $\mathrm{PGE}_{2}$ is causal in ARDS pathophysiology or represents the consequence of upstream pathophysiological events, as well as the unknown translatability of experimentally induced ARDS in rodents to the human COVID-related acute lung injury / ARDS. Findings of Aso (Aso et al., 2012) in human pulmonary microvascular endothelial cells suggest that $\mathrm{PGE}_{2}$ may play an upstream role in the inflammatory cascade by increasing IL-8 release and COX-2 expression, while leaving constitutive COX-1 expression unaffected. Furthermore, in lung tissue from human organ donors, TNFa induced inhibition of surfactant synthesis was caused by $\mathrm{NO}$ generation secondary to $\mathrm{PGE}_{2}$ production, and the TNF $\alpha$ effect could be inhibited by indomethacin (Vara et al., 1996). In addition, a hypothesis has been postulated that reactive oxygen and nitrogen species may contribute to ARDS and other symptoms in viral diseases (Akaike, 2001; Davis and Matalon, 2001; Vielma et al., 2014; Khomich et al., 2018) and some indications of this in animal studies of viral infection have been observed (Nakamura et al., 2002; Kash and Taubenberger, 2015).

Reviews and meta-analyses of randomized controlled studies of various approaches against ARDS in humans have so far generally failed to demonstrate favorable drug effects (Adhikari et al., 2004; Duggal et al., 2015; Lewis et al., 2019), including trials with methylprednisolone, statins, indomethacin, $\mathrm{PGE}_{1}$ and the antioxidant $\mathrm{n}$-acetylcysteine. Intravenous selenium administration although demonstrated some anti-inflammatory action failed to show beneficial effects on overall survival and and ventilation free days in critically ill patients with ARDS (Mahmoodpoor et al., 2019). As corticosteroids and indomethacin have multiple effects on the inflammatory cascade, it is possible that they also inhibit favorable effects of inflammation; a more selective intervention, reducing $\mathrm{PGE}_{2}$ only, may leave beneficial effects of inflammation intact. Nevertheless, there is also some evidence that inhibiting $\mathrm{PGE}_{2}$ might be detrimental, e.g. resulting in impaired bacterial killing of P. aeruginosa (Aoyagi et al., 2017) 
exacerbation of OA-induced ARDS by indomethacin in rats (Sharma et al., 2016) and exacerbation of lung neutrophilic inflammation (Felton et al., 2018).

\section{Sonlicromanol: a clinical trial stage drug compound selectively inhibiting mPGES-1}

Sonlicromanol (KH176; IUPAC chemical name (S)-6-hydroxy-2,5,7,8-tetramethyl-N-((R)-piperidin-3$\mathrm{yl}$ )chroman-2-carboxamide hydrochloride) is a clinical trial stage drug compound (phase 1 completed, phase 2a completed, phase 2b ongoing) (Koene et al., 2017; Beyrath et al., 2018; Janssen et al., 2019) with a triple mode of action (radical trapping, ferroptosis inhibition by inhibiting lipid peroxidation, anti-inflammatory inhibiting mPGES-1). Important in this context is that both the parent compound (sonlicromanol) and its metabolite $(\mathrm{KH} 176 \mathrm{~m})$ are both active and, in humans, $\sim 50 \%$ of the parent compound is converted to the active metabolite KH176m (Koene et al., 2017; Janssen et al., 2019).

We recently investigated the effect of $\mathrm{KH} 176 \mathrm{~m}$ on $\mathrm{PGE}_{2}$ biosynthesis in human control primary fibroblast cells, as well as in the mouse macrophage-like cell line RAW264.7 (Smeitink JAM, Beyrath JD, WO2019101826 - Compounds as MPGES-1 inhibitors PCT/EP2018/082146). Our data indicate that $\mathrm{KH} 176 \mathrm{~m}$ could selectively block the production of $\mathrm{PGE}_{2}$ induced by the inflammatory stimuli lipopolysaccharide (LPS) or interleukin-1 beta (IL-1 $\beta$ ) across species, without showing an effect on the other prostaglandins. We further demonstrated that the inhibitory effect of $\mathrm{KH} 176 \mathrm{~m}$ on $\mathrm{PGE}_{2}$ production is dependent on MPGES-1 inhibition, further blocking mPGES-1 transcriptional expression. Therefore, in addition to it being a novel therapeutic option for mitochondrial disease patients, our results indicate that $\mathrm{KH} 176 \mathrm{~m}$, as well as its parent compound sonlicromanol, may also potentially be used to treat $\mathrm{PGE}_{2}$-driven inflammatory consequences such as might underly the COVID-19 associated ARDS (Figure 2), or when administered early after diagnosis, might prevent progression to ARDS. Sonlicromanol, in addition to its anti-inflammatory properties, also shows stoichiometric radical scavenging activity and prevents lipid peroxidation and thereby lipid ROS driven cell death called ferroptosis (Beyrath et al., 2018). Considering that elevated ROS were detected in plasma and lung tissue of patients with ARDS and their levels correlated with severity of the disease (Castillo et al., 2015) ferroptosis might be a part of pathology in this condition (Castillo et al., 2015).

We suggest to execute a powered investigator-initiated randomized parallel design (ideally placebocontrolled) trial with a treatment duration of 20 days in 30 COVID-19 ICU-admitted patients receiving $50 \mathrm{mg}$ bid oral sonlicromanol, 30 COVID-19 patients receiving $100 \mathrm{mg}$ bid oral sonlicromanol and 30 control patients as comparison, based on currently available medication. This trial will have $80 \%$ power to observe a $50 \%$ reduction in mortality, if mortality would be $80 \%$ without treatment. Note that on 29 March 2020 in the Netherlands, a total 141 of 1248 patients died while in ICU and a total of 35 of 1248 recovered in ICU [source: https://www.stichting-nice.nl accessed April 1st, 2020). The daily ratio of recovering/dying patients in the last week ranges from 0 to 0.38 , with an average of 0.27 (1 discharged vs 3.6 deaths) in the last week. If these numbers are extrapolated, the expected mortality is higher than $50 \%$. Should mortality in the control group be closer to general ARDS estimates (Duggal et al., 2015) e.g. 50\%, the trial will have $40 \%$ power to detect a reduction of $50 \%$ in mortality. Alternatively, early treatment immediately after diagnosis might prevent progression to ARDS, a hypothesis that would require a differently designed study. Finally, we recently observed that the 
active metabolite of sonlicromanol $(\mathrm{KH} 176 \mathrm{~m})$ is able to increase the potency of indomethacin (COX inhibitor) and celecoxib (COX-2 inhibitor) from micromolar to nanomolar range, in an LPS-induced acute inflammation model (unpublished results). Based on the observed synergistic effect we speculate that combination treatment of $\mathrm{KH} 176 \mathrm{~m}$ and a below-normal dose of COX inhibitors may attenuate the side effects caused by COX inhibitors, may produce an equivalent inhibition of $\mathrm{PGE}_{2}$ and might have a synergistic anti-inflammatory response.

We are already exploring the possibility of producing more study drug at short notice to allow an increase in sample size. This would allow increases in sample size; e.g. to detect a decrease of $25 \%$ in mortality and, assuming $80 \%$ mortality, the sample size would be $86 /$ group, nearly identical to samples sizes of the largest outcome studies on mortality with corticosteroids in ARDS (Lewis et al., 2019).

It should be acknowledged (and be clearly conveyed to the appropriate medical ethical board and health authority when asking for trial approval and any patients to be enrolled) that no preclinical or clinical information is currently available about the efficacy or safety of sonlicromanol in (COVID) virus induced ARDS models. Recently, FDA and EMA have shown flexibility in decision making in view of the severity of the situation (e.g. in the rapid approval of diagnostic tests) and have issued guidance on the development of vaccines (https://www.fda.gov/news-events/fda-meetings-conferences-andworkshops/summary-fda-ema-global-regulators-meeting-data-requirements-supporting-first-

human-clinical-trials). The guidance indicates a desire for availability of preclinical data with putative vaccines that demonstrate a potential beneficial effect as well as an absence of worsening of infection, to support first in human trials. Although formally only covering vaccine development, as discussed above, there are both efficacy and safety concerns with anti-inflammatory and antioxidant strategies for ARDS, so that extension of this recommendation to the KH176 situation seems logical. At the same time no animal model for COVID-19 appears to fully reflect the course and/or symptoms of human COVID-19 infection (Martine Denis et al., 2020) and intra-species differences in mPGES-1 have complicated the (preclinical) development of effective human mPGES-1 inhibitors (Ding et al., 2018). Interestingly preclinical data show that $\mathrm{KH} 176 \mathrm{~m}$ is inhibiting mPGES-1 in both human and mouse cells (unpublished).

In clinical trials, sonlicromanol at an oral dose of $100 \mathrm{mg}$ b.i.d. has, thus far, showed an acceptable safety profile, with CNS adverse events and QTC prolongation as dose-limiting adverse events at single doses of 800 and $2000 \mathrm{mg}$ in healthy volunteers (Koene et al., 2017). However, it should be acknowledged that it is unknown whether sonlicromanol might worsen ARDS symptomatology or lead to other unwanted side effects in ARDS. In addition, it should be acknowledged that if sonlicromanol turns out to be efficacious and safe in ARDS, the upscaling of GMP production will take time and effort.

\section{Conclusion and call for experts}

Since its first description more than 50 years ago, ARDS mortality rates remain high. The recent experience in COVID-19 calls for alternative strategies. We here formulate a new hypothesis "lipid mediators play a crucial role in COVID-19 disease progressing and are amenable to treatment intervention". Based on unpublished data and literature searches we propose that selective inhibition of mPGES-1 might serve as an alternative, so far unexplored, treatment option to prevent or counteract COVID-19 ARDS. 
In line with van de Veerdonk et al. (Veerdonk et al., 2020) in their kinins and cytokines hypothesis manuscript recently published, we are inviting experts in the field of corona viruses to test our compound in-vitro and in-vivo. We are actively searching for big-pharma partners willing to assist us in evaluating our hypothesis and the potential of bringing sonlicromanol to COVID-19 patients.

\section{References}

Adhikari NK, Burns KE, Meade MO, Ratnapalan M. Pharmacologic therapies for adults with acute lung injury and acute respiratory distress syndrome. Cochrane Database Syst. Rev. 2004

Agard M, Asakrah S, Morici LA. PGE2 suppression of innate immunity during mucosal bacterial infection. Front. Cell. Infect. Microbiol. 2013; 4: 1-11.

Akaike T. Role of free radicals in viral pathogenesis and mutation. Rev. Med. Virol. 2001; 11: 87-101.

Angelidis I, Simon LM, Fernandez IE, Strunz M, Mayr CH, Greiffo FR, et al. An atlas of the aging lung mapped by single cell transcriptomics and deep tissue proteomics [Internet]. Nat. Commun. 2019; 10: 1-17.Available from: http://dx.doi.org/10.1038/s41467-019-08831-9

Aoyagi T, Newstead MW, Zeng X, Nanjo Y, Peters-Golden M, Kaku M, et al. Interleukin-36y and IL-36 receptor signaling mediate impaired host immunity and lung injury in cytotoxic Pseudomonas aeruginosa pulmonary infection: Role of prostaglandin E2. PLoS Pathog. 2017; 13: 1-27.

Aso $\mathrm{H}$, Ito S, Mori A, Morioka M, Suganuma N, Kondo M, et al. Prostaglandin e 2 enhances interleukin-8 production via ep4 receptor in human pulmonary microvascular endothelial cells. Am. J. Physiol. - Lung Cell. Mol. Physiol. 2012; 302: 266-273.

Bähr I, Spielmann J, Quandt D, Kielstein H. Obesity-Associated Alterations of Natural Killer Cells and Immunosurveillance of Cancer. Front. Immunol. 2020; 11

Bergqvist F, Morgenstern R, Jakobsson PJ. A review on mPGES-1 inhibitors: From preclinical studies to clinical applications [Internet]. Prostaglandins Other Lipid Mediat. 2020; 147: 106383.Available from: https://doi.org/10.1016/j.prostaglandins.2019.106383

Beyrath J, Pellegrini M, Renkema H, Houben L, Pecheritsyna S, Van Zandvoort P, et al. KH176 Safeguards Mitochondrial Diseased Cells from Redox Stress-Induced Cell Death by Interacting with the Thioredoxin System/Peroxiredoxin Enzyme Machinery. Sci. Rep. 2018

Castillo RL, Carrasco Loza R, Romero-Dapueto C. Pathophysiological Approaches of Acute Respiratory Distress syndrome: Novel Bases for Study of Lung Injury. [Internet]. Open Respir. Med. J. 2015; 9: 8391. [cited 2020 Apr 10] Available from: http://www.ncbi.nlm.nih.gov/pubmed/26312099

Chen Y, Guo Y, Pan Y, Zhao ZJ. Structure analysis of the receptor binding of 2019-nCoV. Biochem. Biophys. Res. Commun. 2020

Coulombe F, Jaworska J, Verway M, Tzelepis F, Massoud A, Gillard J, et al. Targeted prostaglandin E2 inhibition enhances antiviral immunity through induction of type I interferon and apoptosis in macrophages. Immunity 2014

Davis I, Matalon S. Reactive Species in Viral Pneumonitis: Lessons From Animal Models. News Physiol. Sci. 2001; 16: 185-190.

Ding K, Zhou Z, Hou S, Yuan Y, Zhou S, Zheng X, et al. Structure-based discovery of mPGES-1 inhibitors 
suitable for preclinical testing in wild-type mice as a new generation of anti-inflammatory drugs. Sci. Rep. 2018

Duggal A, Ganapathy A, Ratnapalan M, Adhikari NKJ. Pharmacological treatments for acute respiratory distress syndrome: Systematic review. Minerva Anestesiol. 2015

Fain JN, Kanu A, Bahouth SW, Cowan GSM, Hiler ML, Leffler CW. Comparison of PGE2, prostacyclin and leptin release by human adipocytes versus explants of adipose tissue in primary culture. Prostaglandins Leukot. Essent. Fat. Acids 2002; 67: 467-473.

Felton JM, Duffin R, Robb CT, Crittenden S, Anderton SM, Howie SEM, et al. Facilitation of IL-22 production from innate lymphoid cells by prostaglandin E2 prevents experimental lung neutrophilic inflammation. Thorax 2018: 1081-1084.

Ferner RE, Aronson JK. Chloroquine and hydroxychloroquine in covid-19 Use of these drugs is premature and potentially harmful [Internet]. 2020; 1432: 9-10.Available from:

http://dx.doi.org/doi:10.1136/bmj.m1432

Full F, Gack MU. Prostaglandin E2: The villain in the host response to influenza virus [Internet]. Immunity 2014; 40: 453-454. [cited 2020 Apr 10] Available from:

http://www.ncbi.nlm.nih.gov/pubmed/24745327

Gomez I, Foudi N, Longrois D, Norel X. The role of prostaglandin E2 in human vascular inflammation. Prostaglandins Leukot. Essent. Fat. Acids 2013

Gonçalves-De-Albuquerque CF, Burth P, Silva AR, De Moraes IMM, De Jesus Oliveira FM, Santelli RE, et al. Oleic acid inhibits lung $\mathrm{Na} / \mathrm{K}$-ATPase in mice and induces injury with lipid body formation in leukocytes and eicosanoid production. J. Inflamm. (United Kingdom) 2013; 10: 1-10.

González-Périz A, Clària J. Resolution of adipose tissue inflammation. ScientificWorldJournal. 2010

Gross S, Tilly P, Hentsch D, Vonesch JL, Fabre JE. Vascular wall-produced prostaglandin E2 exacerbates arterial thrombosis and atherothrombosis through platelet EP3 receptors. J. Exp. Med. 2007; 204: 311-320.

Guo YR, Cao QD, Hong ZS, Tan YY, Chen SD, Jin HJ, et al. The origin, transmission and clinical therapies on coronavirus disease 2019 (COVID-19) outbreak - an update on the status. Mil. Med. Res. 2020

Hara S, Kamei D, Sasaki Y, Tanemoto A, Nakatani Y, Murakami M. Prostaglandin E synthases: Understanding their pathophysiological roles through mouse genetic models [Internet]. Biochimie 2010; 92: 651-659.Available from: http://dx.doi.org/10.1016/j.biochi.2010.02.007

Harmer D, Gilbert M, Borman R, Clark KL. Quantitative mRNA expression profiling of ACE 2, a novel homologue of angiotensin converting enzyme. FEBS Lett. 2002; 532: 107-110.

Ikeda-Matsuo Y, Ota A, Fukada T, Uematsu S, Akira S, Sasaki Y. Microsomal prostaglandin E synthase1 is a critical factor of stroke-reperfusion injury [Internet]. Proc. Natl. Acad. Sci. 2006; 103: 1179011795.Available from: http://www.pnas.org/cgi/doi/10.1073/pnas.0604400103

Janssen MCH, Koene S, de Laat P, Hemelaar P, Pickkers P, Spaans E, et al. The KHENERGY Study: Safety and Efficacy of KH176 in Mitochondrial m.3243A>G Spectrum Disorders. Clin. Pharmacol. Ther. 2019

Kalinski P. Regulation of Immune Responses by Prostaglandin E 2 . J. Immunol. 2012 
Kash JC, Taubenberger JK. The role of viral, host, and secondary bacterial factors in influenza pathogenesis [Internet]. Am. J. Pathol. 2015; 185: 1528-1536.Available from:

http://dx.doi.org/10.1016/j.ajpath.2014.08.030

Khomich OA, Kochetkov SN, Bartosch B, Ivanov A V. Redox biology of respiratory viral infections. Viruses 2018; 10

Koene S, Spaans E, Van Bortel L, Van Lancker G, Delafontaine B, Badilini F, et al. KH176 under development for rare mitochondrial disease: A first in man randomized controlled clinical trial in healthy male volunteers. Orphanet J. Rare Dis. 2017

Kritas S, Ronconi G, Caraffa A, Gallenga C, Ross R, Conti P. Mast cells contribute to coronavirusinduced inflammation: new anti-inflammatory strategy. J. Biol. Regul. Homeost. Agents 2019; 34

Lewis SR, Pritchard MW, Thomas CM, Smith AF. Pharmacological agents for adults with acute respiratory distress syndrome. Cochrane Database Syst. Rev. 2019

Mahmoodpoor A, Hamishehkar H, Shadvar K, Ostadi Z, Sanaie S, Saghaleini SH, et al. The Effect of Intravenous Selenium on Oxidative Stress in Critically III Patients with Acute Respiratory Distress Syndrome [Internet]. Immunol. Invest. 2019; 48: 147-159. [cited 2020 Apr 10] Available from: http://www.ncbi.nlm.nih.gov/pubmed/30001171

Martine Denis, Insights T, Paper L. COVID-19 Overview of information available to support the development of medical countermeasures and interventions against COVID-19. 2020: 1-79.

McCarthy MK, Weinberg JB. Eicosanoids and respiratory viral infection: Coordinators of inflammation and potential therapeutic targets. Mediators Inflamm. 2012

Meng F you, Gao W, Ju Y nan. Parecoxib reduced ventilation induced lung injury in acute respiratory distress syndrome. BMC Pharmacol. Toxicol. 2017; 18: 1-9.

Miller SB. Prostaglandins in Health and Disease: An Overview [Internet]. Semin. Arthritis Rheum. 2006; 36: 37-49. [cited 2017 Aug 25] Available from:

http://linkinghub.elsevier.com/retrieve/pii/S0049017206000497

Mocarski ES. Virus self-improvement through inflammation: No pain, no gain. Proc. Natl. Acad. Sci. U. S. A. 2002; 99: 3362-3364.

Morris P, Papadakos P, Russell J, Wunderink R, Schuster D, Truwit J, et al. A double-blind placebocontrolled study to evaluate the safety and efficacy of L-2-oxothiazolidine-4-carboxylic acid in the treatment of patients with acute respiratory distress syndrome. Crit. Care Med. 2008; 36: 782-788.

Nakamura H, Tamura S, Watanabe I, Iwasaki T, Yodoi J. Enhanced resistancy of thioredoxintransgenic mice against influenza virus-induced pneumonia. Immunol. Lett. 2002; 82: 165-170.

Nakanishi M, Gokhale V, Meuillet EJ, Rosenberg DW. mPGES-1 as a target for cancer suppression: A comprehensive invited review \&quot;Phospholipase A2 and lipid mediators\&quot;. [Internet]. Biochimie 2010; 92: 660-4.[cited 2017 Feb 13] Available from: http://www.ncbi.nlm.nih.gov/pubmed/20159031

Osborn O, Gram H, Zorrilla E, Conti B, Bartfai T. Insights into the roles of the inflammatory mediators IL-1, IL-18 and PGE2 in obesity and insulin resistance. Swiss Med. Wkly. 2008; 138: 665-673.

Pace S, Rossi A, Krauth V, Dehm F, Troisi F, Bilancia R, et al. Sex differences in prostaglandin biosynthesis in neutrophils during acute inflammation. Sci. Rep. 2017; 7: 1-10. 
Park JH, Park EB, Lee JY, Min JY. Identification of novel membrane-associated prostaglandin e synthase-1 (mPGES-1) inhibitors with anti-influenza activities in vitro. Biochem. Biophys. Res. Commun. 2016; 469: 848-855.

Park JY, Pillinger MH, Abramson SB. Prostaglandin E2 synthesis and secretion: The role of PGE2 synthases [Internet]. Clin. Immunol. 2006; 119: 229-240.Available from: http://linkinghub.elsevier.com/retrieve/pii/S1521661606000453

Pawelzik SC, Avignon A, Idborg H, Boegner C, Stanke-Labesque F, Jakobsson PJ, et al. Urinary prostaglandin D2 and E2 metabolites associate with abdominal obesity, glucose metabolism, and triglycerides in obese subjects. Prostaglandins Other Lipid Mediat. 2019; 145

Pierre C, Guillebaud F, Airault C, Baril N, Barbouche R, Save E, et al. Invalidation of Microsomal Prostaglandin E Synthase-1 (mPGES-1) Reduces diet-induced low-grade inflammation and adiposity. Front. Physiol. 2018

Pritze S, Peskar BA, Simmet T. Release of eicosanoids and endothelin in an experimental model of adult respiratory distress syndrome. In: Agents and Actions. 1992.

Ricciotti E, Fitzgerald GA. Prostaglandins and inflammation. Arterioscler. Thromb. Vasc. Biol. 2011

Rice TW, Wheeler AP, Thompson BT, deBoisblanc BP, Steingrub J, Rock P, et al. Enteral omega-3 fatty acid, gamma-linolenic acid, and antioxidant supplementation in acute lung injury. [Erratum appears in JAMA. 2012 Feb 8;307(6):563]. Jama 1574

Riendeau D, Aspiotis R, Ethier D, Gareau Y, Grimm EL, Guay J, et al. Inhibitors of the inducible microsomal prostaglandin E2 synthase (mPGES-1) derived from MK-886 [Internet]. Bioorganic Med. Chem. Lett. 2005; 15: 3352-3355. [cited 2017 Apr 6] Available from:

http://www.ncbi.nlm.nih.gov/pubmed/15953724

Rodriguez-Morales AJ, Cardona-Ospina JA, Gutiérrez-Ocampo E, Villamizar-Peña R, Holguin-Rivera Y, Escalera-Antezana JP, et al. Clinical, laboratory and imaging features of COVID-19: A systematic review and meta-analysis. Travel Med. Infect. Dis. 2020

Sander WJ, O’Neill HG, Pohl CH. Prostaglandin E2 as a modulator of viral infections. Front. Physiol. 2017

Sharma P, Pandey R, Deshpande S. Indomethacin Exacerbates Oleic Acid-Induced Acute Respiratory Distress Syndrome in Adult Rats. Indian J. Physiol. Pharmacol. 2016; 60: 82-89.

Smith WL, Urade Y, Jakobsson PJ. Enzymes of the cyclooxygenase pathways of prostanoid biosynthesis. Chem. Rev. 2011; 111: 5821-5865.

Sreeramkumar V, Fresno M, Cuesta N. Prostaglandin e 2 and T cells: Friends or foes. Immunol. Cell Biol. 2012; 90: 579-586.

Sungnak W, Huang N, Bécavin C, Berg M, Network HCALB. SARS-CoV-2 Entry Genes Are Most Highly Expressed in Nasal Goblet and Ciliated Cells within Human Airways [Internet]. 2020Available from: http://arxiv.org/abs/2003.06122

Tron GC, Pirali T, Sorba G, Pagliai F, Busacca S, Genazzani A a. Microsomal Prostaglandin E2 Synthase1 (mPGES-1): A Novel Anti-Inflammatory Therapeutic Target Richard. 2006; 51: 1-12.

Tsuge K, Inazumi T, Shimamoto A, Sugimoto Y. Molecular mechanisms underlying prostaglandin E2exacerbated inflammation and immune diseases. Int. Immunol. 2019; 31: 597-606. 
Vara E, Arias-Díaz J, García C, Hernández J, Balibrea JL. TNF- $\alpha$-induced inhibition of PC synthesis by human type Il pneumocytes is sequentially mediated by PGE2 and NO. Am. J. Physiol. - Lung Cell. Mol. Physiol. 1996

Veerdonk F van de, Netea MG, Deuren M van, Meer JWM van der, Mast Q de, Bruggemann RJ, et al. Kinins and Cytokines in COVID-19: A Comprehensive Pathophysiological Approach. 2020

Vielma J, Bonilla E, Chacín-Bonilla L, Mora M, Medina-Leendertz S, Bravo Y. Effects of melatonin on oxidative stress, and resistance to bacterial, parasitic, and viral infections: A review. Acta Trop. 2014; 137

Wu D, Mura C, Beharka AA, Han SN, Paulson KE, Hwang D, et al. Age-associated increase in PGE2 synthesis and COX activity in murine macrophages is reversed by vitamin E. Am. J. Physiol. - Cell Physiol. 1998; 275

Xu H, L. Z, J. D, J. P, H. D, X. Z, et al. High expression of ACE2 receptor of 2019-nCoV on the epithelial cells of oral mucosa. Int. J. Oral Sci. 2020

Yao C, Narumiya S. Prostaglandin-cytokine crosstalk in chronic inflammation. Br. J. Pharmacol. 2019

Yu H, Ni YN, Liang ZA, Liang BM, Wang Y. The effect of aspirin in preventing the acute respiratory distress syndrome/acute lung injury: A meta-analysis. Am. J. Emerg. Med. 2018; 36: 1486-1491.

Zhao J, Zhao J, Perlman S. T Cell Responses Are Required for Protection from Clinical Disease and for Virus Clearance in Severe Acute Respiratory Syndrome Coronavirus-Infected Mice. J. Virol. 2010; 84: 9318-9325.

Zhao Jincun, Zhao Jingxian, Legge K, Perlman S. Age-related increases in PGD 2 expression impair respiratory DC migration, resulting in diminished $T$ cell responses upon respiratory virus infection in mice. J. Clin. Invest. 2011; 121: 4921-4930.

\section{Potential conflict of Interest:}

$\mathrm{JS}$ is the founding CEO, RVM the CMO, JB the CSO and XJ, SP and HR researchers at Khondrion BV, a clinical stage pharma-company with its primary focus on mitochondrial disease. Sonlicromanol is patented by the Khondrion IP-BV.

\section{Acknowledgement:}

The authors would like to thank the other members of the Khondrion Team for continuous support. 


\section{Box 1: The Solidarity Trial}

Remdesivir was previously tested as an Ebola treatment. It has generated promising results in animal studies for Middle East Respiratory Syndrome (MERS-CoV) and severe acute respiratory syndrome (SARS), which are also caused by coronaviruses, suggesting it may have some effect in patients with COVID-19.

Lopinavir/Ritonavir is a licensed treatment for HIV. Evidence for COVID-19, MERS and SARS is yet to show it can improve clinical outcomes or prevent infection. This trial aims to identify and confirm any benefit for COVID-19 patients. While there are indications from laboratory experiments that this combination may be effective against COVID-19, studies done so far in COVID-19 patients have been inconclusive.

Interferon beta-1a is used to treat multiple sclerosis.

Chloroquine and hydroxychloroquine are very closely related and used to treat malaria and rheumatology conditions respectively. In China and France, small studies provided some indications of possible benefit of chloroquine phosphate against pneumonia caused by COVID-19 but need confirmation through randomized trials.

\section{Box 2: The Discovery Trial}

The DISCOVERY trial includes five treatment modalities:

- standard of care

- standard of care plus remdesivir

- standard of care plus lopinavir and ritonavir,

- standard of care plus lopinavir, ritonavir and interferon-beta

- standard of care plus hydroxychloroquine.

Allocation of patients to the various treatment modalities will be randomized, i.e. by random draw, but patients and physicians will know which treatment is used (this is called an open trial). The analysis of treatment efficacy and safety will be evaluated 15 days after inclusion of each patient. 


\section{Figure Legends}

Figure 1: The prostaglandins biosynthesis pathway and selective MPGES1 inhibition.

Following inflammatory stimuli activation of the arachidonic pathway leads to the production of prostaglandins. COX2 and mPGES-1 are inducible enzymes and their expression is increased in inflamed tissues. A general decrease in prostaglandin species occurs following inhibition at the level of the COX 2 enzyme by NSAIDs and COXibs (COX inhibitors). Sonlicromanol metabolite selectively blocks the activity of the microsomal prostaglandin E synthase-1. Consequently, this leads to specific lowering of the $\mathrm{PGE}_{2}$ and thereby counteracting its effect. As opposed to upstream COX inhibition, selective $\mathrm{mPGES}-1$ inhibition still permits basal PGE2 production via its constitutive enzymes CPGES and mPGES-2. sPLA: soluble phospholipase A; COX: cyclooxygenase; PG: prostaglandin; Tx:

tromboxane

\section{Figure 2: Covid-19, Lung failure and PGE $_{2}$. A multi-faceted pathophysiological cascade}

The expression of the ACE-2 receptor is ubiquitous. Within the upper- and lower respiratory tract expression is highest in nasal goblet and ciliated cells (Sungak et al., 2020). Within the nasal cavity and submucosal respiratory tract mucosal mast cells are the most important first line of defense. The mechanism how cells respond to viruses is cell-type dependent. While nasal goblet and ciliated cells respond with viral death mechanisms, mast cells act with early and late inflammatory responses. Both PAMPS and DAMPS may cause an increase in lipid mediators of which $\mathrm{PGE}_{2}$ production can be selectively decreased by microsomal prostaglandin E synthase-1 inhibitors like sonlicromanol. Additionally, alveolar macrophages are important sentinels in respiratory infection and participate in the activation of innate and adaptive host immune response. $\mathrm{mPGES}$-1-derived PGE2 is known to inhibit this response therefore its inhibition could enhance host immune response against the viral infection. As COVID-19 associated pathology is multi-faceted the application of anti-inflammatory cytokines might cause a synergistic effect. Ageing and pre-existing conditions like obesity are associated with more severe outcome. The increased inflammation status observed in obesity might be one of the underlying contributing factors making obese patients more inflammation prone. 
Figure 1: The prostaglandins biosynthesis pathway and selective mPGES1 inhibition.

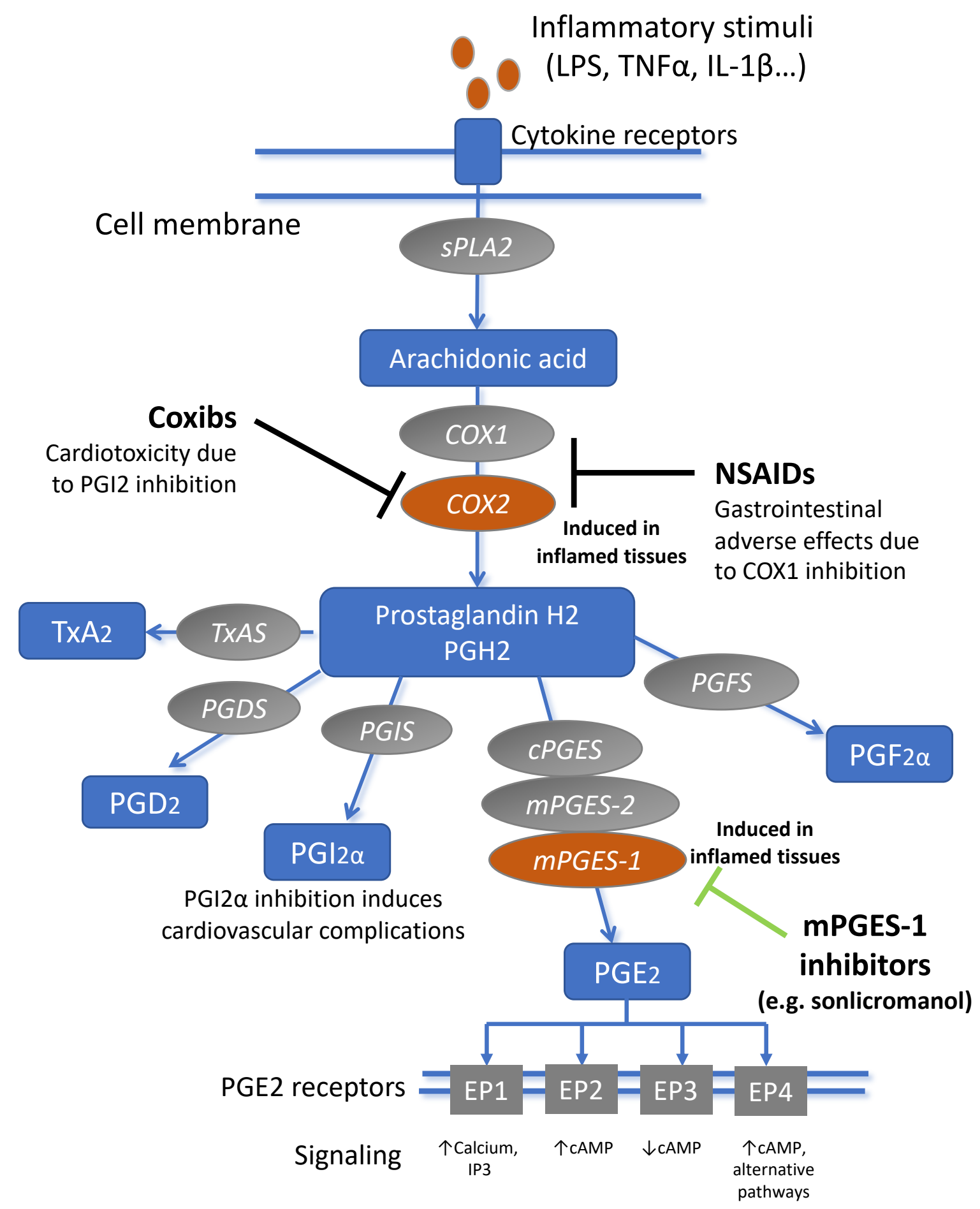


Figure 2: Covid-19, Lung failure and $\mathrm{PGE}_{2}$. A multi-faceted pathophysiological cascade

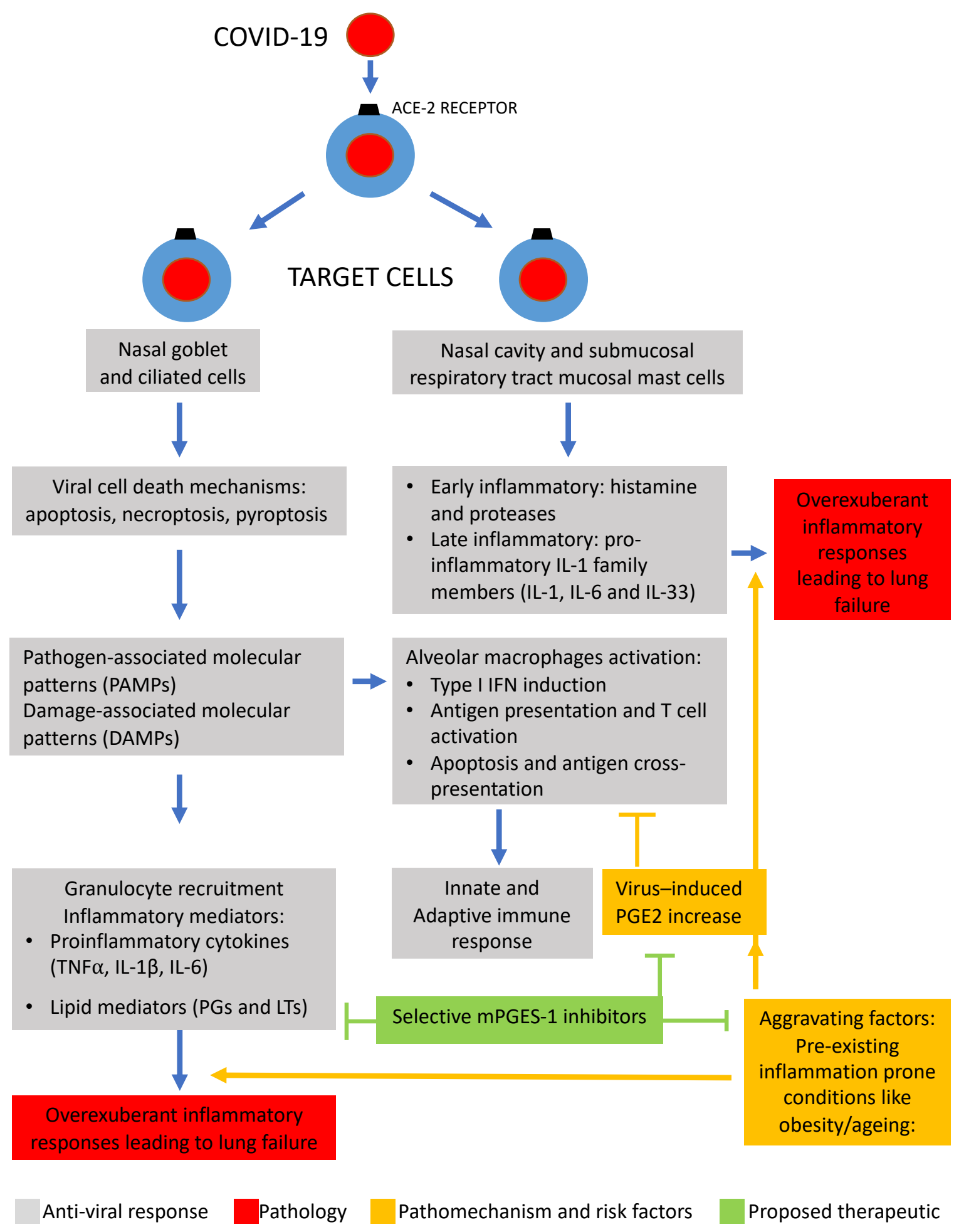


